\title{
La Inteligencia Social: Aportes Desde su Estudio en Niños y Adolescentes con Altas Capacidades Cognitivas
}

\section{Social Intelligence: Contributions From its Study in Children and Adolescents With High Cognitive Abilities}

\author{
Verónica López \\ Universidad Autónoma de Madrid \\ Universidad Alberto Hurtado
}

\begin{abstract}
El constructo de "inteligencia social" ha suscitado reiterados cuestionamientos a lo largo de la historia de la Psicología. Una forma de contribuir al debate es a través del estudio del razonamiento social en personas con altas capacidades cognitivas. Las puntuaciones de 50 niños y adolescentes con altas capacidades cognitivas en dos medidas de razonamiento social fueron comparadas con las de un grupo de pares de capacidad cognitiva media equiparados uno a uno en sexo y edad cronológica. Los resultados indican que estos niños y adolescentes presentan un rendimiento significativamente superior a sus pares en tareas relacionadas con la inteligencia social, destacando sobre todo en procesos de razonamiento que involucran la comprensión y definición del problema social, la planificación de estrategias y la anticipación de consecuencias sociales.
\end{abstract}

Palabras Clave: inteligencia, inteligencia social, altas capacidades cognitivas, talento académico.

\begin{abstract}
The construct of "social intelligence" has aroused reiterated debates throughout the history of Psychology. One way to contribute to this debate is through the study of social reasoning in people with high cognitive abilities. The scores of 50 Spanish-speaking children and adolescents with high cognitive abilities in two measures of social reasoning were compared to age- and sex-matched peers of normotypical cognitive ability. The results indicate that high-ability children and adolescents present a significatively superior performance compared to their peers in tasks related with social intelligence, especially in reasoning processes which involve understanding and defining the social problem, planning social strategies and anticipating social consequences.
\end{abstract}

Keywords: intelligence, social intelligence, gifted, high cognitive abilities, academic giftedness.

\section{La Inteligencia: Reseña Histórica}

La medición de la inteligencia como cualidad individual o "facultad mental" debe sus inicios a Binet y Simon (Binet, Simon \& Kite, 1916). Spearman (1927) fue el primero en proponer una teoría monolítica de la inteligencia humana, entendida como una capacidad general que permanece estable a lo largo de la vida: “ $G$ '. Spearman $(1904,1927)$ observó que muchas tareas de distintas pruebas de inteligencia correlacionaban entre sí. Esto lo llevó a plantear la existencia de un factor general de inteligencia. Hoy la idea de una capacidad cognitiva general sigue siendo defendida por muchos investigadores (Jensen, 2002; Lubinski, 2004).

Verónica López Leiva, Escuela de Psicología y Facultad de Educación, Universidad Alberto Hurtado.

Los resultados informados en este artículo forman parte de la tesis doctoral desarrollada por la autora en la Facultad de Psicología de la Universidad Autónoma de Madrid, con el apoyo de la Beca Presidente de la República para Estudios en el Extranjero.

La correspondencia relativa a este artículo deberá ser dirigida al e-mail: velopez@uahurtado.cl
La propuesta de una inteligencia general, estable e inmodificable no fue aceptada por todos. Algunos investigadores perfeccionaron las técnicas estadísticas que se estaban desarrollando en el momento para rebatir a Spearman. Thurstone (1938), por ejemplo, planteó que el factor general propuesto por Spearman era solo una de muchas posibilidades de agrupación de los datos bajo el análisis factorial. Mediante la rotación de los ejes, Thurstone encontró siete factores que consideró capacidades mentales con igual nivel de importancia.

Guilford (1967), desde una posición neofactorialista, planteó el Modelo de la Estructura del Intelecto. Para él, la inteligencia estaría compuesta por una multitud de habilidades agrupadas en tres dimensiones distintas: Operaciones, contenidos y productos, que combinadas daban lugar a 120 habilidades intelectuales específicas. Guilford (1967) fue uno de los primeros en plantear la existencia de otras aptitudes o capacidades no relacionadas con lo lógico-matemático, lo verbal o lo viso-espacial, tales como la creatividad, el pensamiento divergente $y$, como veremos más adelante, la inteligencia social. 
Luego del auge por medir los componentes de la inteligencia haciendo uso de técnicas psicométricas, las últimas décadas han visto surgir el desarrollo de modelos menos orientados por la psicometría y más fundados teóricamente. Sternberg (1984), por ejemplo, propuso una conceptualización de la inteligencia basada en componentes básicos del procesamiento de la información que permiten explicar las diferencias individuales en la inteligencia exitosa (Sternberg, 2005). Sternberg (1984) entiende a la inteligencia en función de los recursos que tienen las personas para procesar la información y la experiencia. En su Teoría Triárquica de la Inteligencia, Sternberg propuso que la inteligencia está compuesta por tres tipos de habilidades: analíticas, creativas y prácticas.

Desde una posición de mayor apertura conceptual, Gardner (1983) planteó la existencia de inteligencias múltiples, entendiéndolas como entidades nosológicas independientes pero interrelacionadas. Gardner $(1983,2000)$ propuso inicialmente siete, luego nueve tipos de inteligencia: lingüístico-verbal, lógica-matemática, viso-espacial, corporalkinestésica, musical, interpersonal, intrapersonal, naturalista y espiritual. De esta manera, Gardner ha reconceptualizado el constructo de inteligencia desde un enfoque que privilegia las capacidades analíticas, verbales y lógico-formales, a otro que integra habilidades en los ámbitos artísticos y sociales.

\section{La Inteligencia Social}

La concepción y el estudio de la inteligencia social han formado parte del debate general acerca de la inteligencia, aun cuando su estudio ha sido más controversial y menos investigado. A continuación revisamos las concepciones sostenidas a lo largo de la historia de la Psicología sobre el constructo de "inteligencia social", y las maneras en que ésta se ha estudiado.

\section{Concepciones Sobre Inteligencia Social}

En 1920 Thorndike consideraba que la inteligencia se refería a la habilidad de una persona para comprender y manejar a otras personas y para involucrarse en interacciones sociales adaptativas. Como representante de la posición factorialista, Thorndike (1920) propuso que la inteligencia se dividía en tres dimensiones: inteligencia abstracta, inteligencia mecánica e inteligencia social. Estas dimensiones reflejaban la capacidad para compren- der y manejar ideas, objetos concretos y personas, respectivamente.

Vernon (1933) entendió a la inteligencia social como la habilidad para llevarse bien con las personas, el conocimiento de los asuntos sociales, la susceptibilidad a los estímulos provenientes de otros miembros de un grupo, y el insight a los estados de ánimo temporales y a los rasgos de personalidad de las personas que no conoce.

A partir de estos antecedentes, Moss y Hunt (1927) crearon el primer test de inteligencia social, el George Washington Social Intelligence Test. Este instrumento contenía las siguientes tareas: juicios en situaciones sociales, memoria de nombres y caras, observación del comportamiento humano, reconocimiento de los estados mentales que subyacen a las palabras, reconocimiento de los estados mentales que subyacen a las expresiones faciales, información social y sentido del humor (Kihlstrom $\&$ Cantor, 2000). No obstante, la relativamente alta correlación entre este test y algunas medidas de inteligencia general, $r=.54$ (Hunt, 1928) mermaron el interés inicial por la evaluación de la inteligencia social (Thorndike \& Stein, 1937).

A partir de la década de los 40 , el interés de la corriente dominante se centró en la medición del factor "G" propuesto por Spearman (1927), que no incluía un componente social. Más bien, se consideraba que la comprensión de las personas y sus interacciones formaba parte de la capacidad intelectual general. Tampoco Wechsler $(1939 / 1958,1974)$ se interesó por el concepto de inteligencia social, considerando a ésta como una "inteligencia general aplicada a situaciones sociales" (1939/1958, p. 75). Eso sí, Wechsler (1939/1958) ha reconocido que la subtarea de Historias de su test puede servir como una medida de inteligencia social, ya que evalúa la comprensión de situaciones sociales.

A fines de la década de los '60, Guilford (1967) revivió la discusión acerca de la existencia de una inteligencia de tipo social. En su modelo planteó la existencia de 120 que consistían en combinaciones de cinco operaciones, cuatro contenidos y seis productos. Para Guilford la inteligencia social estaría contemplada en los contenidos conductuales. Propuso que éstos consistían en la información implicada en las interacciones entre individuos, donde se consideran las actitudes, necesidades, deseos, estados de ánimo, percepciones, pensamientos, etc., de otros y de nosotros mismos. La habilidad subyacente a este contenido sería la empatía (Rosas, Boetto \& Jordán, 1999). 
A partir de esta teoría, O Sullivan, Guilford y deMille (1965) crearon un test para evaluar la inteligencia social en su dimensión conductual, entendida como la habilidad para juzgar a las personas, y Hendricks, Guilford y Hoepfner (1969) desarrollaron medidas de afrontamiento social (social coping). En ambos casos surgieron dificultades respecto a los criterios de validación interna y externa, y se volvieron a encontrar correlaciones significativas con medidas de inteligencia general. No obstante, los resultados de otros estudios han sugerido una relativa independencia entre ambos tipos de inteligencia, aun cuando la inteligencia social aparezca en sí como multifactorial (Kihlstrom \& Cantor, 2000).

Desde luego, la década de los 90 ha visto un mayor interés por la comprensión, si no medición, de la inteligencia de tipo social. A este interés han contribuido los planteamientos de Gardner (1983) en relación a la existencia de inteligencias múltiples; entre ellas, la inteligencia interpersonal. La inteligencia interpersonal sería aquella involucrada en el entendimiento y en el actuar según la comprensión de las demás personas, e incluye la capacidad de identificar de manera diferencial los estados de ánimo, temperamentos, motivaciones e intenciones de los demás, que pueden expresarse a través de medios verbales y no verbales (Gardner, 1983).

También Sternberg (1984) ha aportado a la conceptualización de una inteligencia social. Según él, la inteligencia práctica consiste en habilidades de resolución de problemas en contextos de la vida cotidiana, e incluye a la inteligencia social (Sternberg \& Wagner, 1986). Sternberg y Barnes (1988) buscaron evaluar la inteligencia social, definiéndola como la capacidad para decodificar de manera precisa la información social. Desde la Teoría Triárquica, sostuvieron que tanto los metacomponentes como los componentes de ejecución y de adquisición de conocimientos subyacen a ésta.

\section{Medición de la Inteligencia Social}

La medición de la inteligencia social no ha estado exenta de dificultades. Las primeras tareas experimentales diseñadas para evaluarla se debatieron entre la evaluación del comportamiento y el razonamiento socialmente inteligente. $\mathrm{Al}$ respecto, las diferencias entre el tipo de ítems que medían conductas (O'Sullivan et al., 1965), y las que medían estrategias de afrontamiento en situaciones sociales (Hendricks et al., 1969), es notoria. Con todo, los primeros tests estuvieron orientados a la medición de productos o resultados (outcomes).

Sternberg y Wagner (1989) extendieron los límites tradicionales de la evaluación de la inteligencia social a través de un tipo de medición que incorporaba el análisis de los procesos cognitivos implicados y la evaluación de interacciones en contextos específicos. Los autores diseñaron una tarea en la que los participantes debían identificar las características o rasgos que las personas utilizaban para juzgar correctamente una figura a partir de una fotografía. Encontraron diferencias individuales en el número de estrategias distintas utilizadas. Además, las personas previamente identificadas como más hábiles socialmente indicaban como respuesta las estrategias que resultaban más eficaces para cada situación.

Candeias (2003), basándose en la Teoría Triárquica, creó la Prueba Cognitiva de Inteligencia Social (Prova Cognitiva de Inteligência Social). Esta tarea está basada en el modelo de Sternberg (1984) y en el modelo de estrategias de negociación interpersonal de Selman (Yeates \& Selman, 1989). El modelo de Selman plantea niveles progresivos de complejidad, que reflejan la capacidad para diferenciar e integrar diferentes puntos de vista: (a) nivel impulsivo: las estrategias se centran en comportamientos físicos-impulsivos orientados a conseguir objetivos inmediatos y materiales; (b) nivel unilateral: búsquedas unilaterales de control o apaciguamiento de otra persona; (c) nivel recíproco: búsquedas por satisfacer las necesidades de ambas personas de manera recíproca, a través de promesas, acuerdos, persuasión, etc.; y (d) nivel colaborativo: búsquedas por modificar los deseos propios o del interlocutor, para conseguir objetivos mutuos.

En definitiva, la propuesta de Sternberg, desarrollada y re-elaborada posteriormente por Candeias, otorga énfasis a la evaluación de la inteligencia social en contextos sociales específicos, así como a la medición de las estrategias ideadas para identificar y afrontar situaciones de carácter interpersonal. Este tipo de evaluación se acerca mucho a los esfuerzos desarrollados en el campo educativo por medir las llamadas competencias socioemocionales. Éstas consisten en la evaluación de las estrategias que los sujetos son capaces de idear para resolver un problema de carácter social (Díaz-Aguado \& Royo, 1995). Un instrumento elaborado para estos fines, el Cuestionario de Interacción con Compañeros (Díaz-Aguado \& Royo, 1995), presenta a los alumnos distintas situaciones escolares entre dos compañeros, en las que hay un conflicto; el 
sujeto debe indicar todas las posibles soluciones al problema y justificar sus respuestas.

De los datos previamente presentados se desprenden algunos asuntos que requieren de mayor estudio. A nivel teórico, no está claro si la inteligencia social corresponde a un sub-tipo de inteligencia general o puede ser considerada una inteligencia "independiente" de ésta. Una posición intermedia y que ha sido adoptada por algunos autores durante la última década (véase Gardner, 1983, 2000; Goleman, 2006) es que se trataría de una capacidad de dominio específico, es decir, que la mente humana estaría altamente predispuesta a desarrollar herramientas para manejar un determinado tipo de contenido, en este caso referido a las personas y sus relaciones. A nivel empírico, persiste el debate respecto a cómo evaluar la inteligencia social (e.g. como procesos o productos). Al respecto, los avances desde la psicología cognitiva que buscan medir las estrategias cognitivas desplegadas para resolver problemas cotidianos específicos, parecen una alternativa viable y fructífera.

\section{Inteligencia Social y Alta Capacidad Cognitiva}

Dentro de este contexto y con el propósito de aportar al debate anterior, resulta interesante evaluar la inteligencia social en una población que tradicionalmente se ha considerado portadora de una "alta G": las personas con altas capacidades cognitivas (ACC). Su estudio en niños y adolescentes con altas capacidades reviste interés pues permite identificar posibles evoluciones en las estrategias desarrolladas para resolver problemas de tipo social.

A continuación presentamos una síntesis sobre la investigación en niños y adolescentes con ACC, así como los escasos datos existentes respecto al estudio de la inteligencia social en personas con altas capacidades ${ }^{1}$.

\section{Características del Desarrollo Cognitivo y Emocional de Niños con Altas Capacidades Cognitivas}

La literatura informa de una serie de características en los ámbitos cognitivos, afectivos y sociales que serían distintivas de los niños y adolescentes que

\footnotetext{
Debido a las limitaciones de espacio, no se discutirán las conceptualizaciones teóricas acerca de la alta capacidad (al respecto véase Bralic \& Romagnoli, 2000; Gagné, 1993; Marland, 1972; Renzulli, 1994; y Sternberg, 1984), sino sólo los antecedentes referidos al estudio del razonamiento social de este grupo.
}

puntúan alto en las pruebas de inteligencia (Casillas, 1996; Freeman, 1998; George, 1992). Éstas no se manifestarían de una forma homogénea en todos los niños y adolescentes sino que se exhibirían agrupadas o en combinación con otras características (George, 1992).

En el ámbito cognitivo, destaca en ellos el uso de estrategias cognitivas y metacognitivas más eficaces. Shore y Kanevsky (1993) distinguen siete características fundamentales: (a) memoria y conocimiento de base, (b) procesos autorreguladores, (c) velocidad en los procesos de aprendizaje, (d) representación de los problemas y categorización, (e) conocimiento procedural, (f) flexibilidad, y (g) preferencia por la complejidad (véase también Jiménez Fernández, 2004). Además, se ha reconocido en este grupo de niños y adolescentes una alta motivación hacia el aprendizaje (Kanevsky, 1994; Perleth \& Heller, 1994; Rogers, 1986; Van Tassel-Baska, 1998; Winner, 2000).

En el ámbito socioafectivo destaca: (a) la asincronía o disincronía evolutiva (un desfase en el desarrollo de las áreas cognitiva, social y afectiva de los niños y adolescentes con altas capacidades cognitivas (Silverman, 1993; Terrasier, 1992), (b) una alta sensibilidad e intensidad emocional (mayor sensibilidad a experiencias personales y acontecimientos históricos que consideran injustos y dolorosos, véase Guignard \& Zenasni, 2004; Morelock, 2000; Piechowski \& Colangelo, 2004; Roeper, 1982), y (c) el uso de estrategias distintivas de afrontamiento social para hacer frente al hecho de sentirse diferente de los demás (Cross \& Coleman, 1993; Cross, Coleman \& Stewart, 1995; Rimm \& Rimm-Kaufman, 2000; Swiatek \& Dorr, 1998).

Si los niños y adolescentes con ACC presentan ventajas en su procesamiento cognitivo, tanto en las etapas iniciales de identificación y representación del problema, como en la resolución de éste a través del uso de estrategias autorreguladoras, quizás estas estrategias se transfieren al dominio social, en aquellas situaciones que requieren del dominio de estrategias de decodificación social, resolución de problemas interpersonales, anticipación de las consecuencias, verificación de la resolución, etc. Quizás, incluso, sean más conscientes de las estrategias cognitivas que emplean en la decodificación y codificación de problemas sociales. A continuación se presentan algunos antecedentes que permiten apoyar estas ideas. 


\section{Alta Capacidad Cognitiva e Inteligencia Social}

Aunque escasos, existen estudios que han relacionado la ACC con el razonamiento social en infantes y en adultos.

Cupertino y Ancona-López (1992) presentaron a niños con ACC de 10 a 12 años viñetas con dos personajes en una situación de interacción social y les pidieron a describir la historia, indicar el perfil de personalidad de los personajes e identificar las expresiones faciales y verbales. Encontraron heterogeneidad en las puntuaciones, lo que los llevó a plantear que la elevada competencia emocional no es una cualidad característica de los niños con ACC. Sin embargo, al no haber grupo control, no es posible sostener que estos niños presenten (o no) mejores estrategias de interacción social que sus pares.

Gross (2000) encontró que los niños con ACC buscaban relaciones de amistad cercanas, estables y basadas en la confianza mutua a una edad cronológica en la que los niños de capacidad habitual solo buscaban compañeros de juego. Los niños de capacidad extrema $(\mathrm{CI}>160)$ comenzaban a buscar relaciones basadas en la aceptación incondicional cuatro a cinco años antes que sus compañeros de igual edad cronológica. Estos resultados podrían estar indicando que los niños con ACC presenten una mayor "edad social", es decir, un nivel de razonamiento social que los sitúa más cercano a sus pares de igual nivel cognitivo que cronológico. Otros antecedentes apoyan esta idea, tales como la búsqueda desde edades muy tempranas de la compañía de niños mayores y con niveles similares de desarrollo intelectual (Hollingworth, 1931; O'Shea, 1960, en Bralic \& Romagnoli, 2000) o con adultos (George, 1992; Terrassier, 1992).

Powell (1980) comparó la adopción de roles sociales con el nivel de razonamiento "post-formal" (desde el enfoque piagetano) de adultos con ACC. Sus resultados confirmaron su hipótesis de una secuencia invariada del razonamiento abstracto así como del razonamiento social: ningún sujeto presentó un nivel característico de adopción de roles de tipo 7 (el más complejo), sin presentar un nivel tipo 6 ; y ningún sujeto presentó una respuesta tipo 6 , sin presentar una respuesta tipo 5). Además, encontró que ningún adulto presentaba el nivel más complejo de adopción de roles ("empatía interactiva") $\sin$ presentar también el nivel más alto de razonamiento formal ("post-formal"). Pero por el contrario, no todas las personas que operaban a nivel post-formal operaban al nivel más alto de razonamiento social.
Lamentablemente, este estudio tampoco contó con grupo control, por lo que no es posible extraer conclusiones respecto a diferencias entre el grupo con ACC y la población general.

Los datos anteriores permiten hipotetizar que las personas con ACC presentarán un nivel adecuado de razonamiento social -ya sea al mismo nivel que sus pares de capacidad cognitiva media, o incluso superior. Interesa estudiar si la alta capacidad cognitiva incide de manera similar en los aspectos propiamente "intelectuales" del razonamiento social (planificación/ejecución/verificación) como en el contenido de las cogniciones sobre situaciones sociales (saber qué hacer ante una situación de conflicto interpersonal).

\section{Método}

Con el objetivo de estudiar algunos componentes de la inteligencia social en niños y adolescentes con ACC se realizó un estudio cuasi-experimental de tipo ex post facto. La hipótesis de estudio era que los niños y adolescentes con ACC presentarían un rendimiento significativamente superior a sus pares en tareas de razonamiento social.

\section{Participantes}

La muestra estuvo conformada por 100 participantes, 50 de ellos con ACC y 50 de capacidad cognitiva media. La capacidad cognitiva fue evaluada a través de la Escala de Inteligencia General de Wechsler en su versión para niños y adolescentes (WISC-R) y preescolares (WWPSI). Los participantes fueron agrupados en función de su capacidad cognitiva (dos niveles: alto - medio) y de su edad cronológica (cinco tramos de edad: 4.0 a 5.11 años; 6.0 a 8.11 años; 9.0 a 11.11 años; 12.0 a 14.11 años; y 15.0 a 17.11 años.

Los alumnos con ACC fueron seleccionados siguiendo un procedimiento de muestreo intencionado. Se contactó a niños y adolescentes de habla hispana, previamente identificados como alumnos con ACC por sus respectivos centros escolares, o por algún centro especializado en el tema ${ }^{2}$. Los participantes del grupo de comparación tenían una puntuación de 130 o más en el "CI Total" de la Escala de Wechsler $(M=136.36 ; D S=4.95)$.

Los participantes del grupo de comparación fueron equiparados uno a uno con los participantes del grupo con ACC en tres aspectos: (a) eran compañeros de curso, (b) eran del mismo sexo, y (c) tenían la misma edad cronológica en años y meses (se aceptó una diferencia de dos meses cronológicos). Además, debían obtener una puntuación en la Escala de Wechsler que los ubicara dentro del rango medio, es decir, dentro de una desviación típica de la media $(M=101.18 ; D S=10.23)$. Se solicitó consentimiento informado a los padres/apoderados de todos los participantes.

\footnotetext{
2 Agradecemos la colaboración del Colegio Santa María la Real de Pamplona (Navarra, España), de la Xunta de A Coruña (Galicia, España), de la Asociación de Niños Superdotados de Granada (Granada, España) y del Programa PENTA-UC (Santiago, Chile). Por razones de organización del sistema escolar (en España la enseñanza es obligatoria sólo hasta los 16 años) los alumnos del grupo de mayor edad, de 16 a 18 años, fueron evaluados en Chile.
} 


\section{Instrumentos}

Prova Cognitiva de Inteligencia Social, PCIS (Candeias, 2003, 2005). Contiene 6 láminas pictóricas que describen una situación en la que participan tres personajes. Tres láminas presentan situaciones sociales bien definidas y las demás presentan situaciones poco estructuradas. En este estudio se utilizaron sólo las láminas con situaciones poco estructuradas para aumentar la complejidad de la situación experimental. La tarea consiste en una entrevista semi-estructurada en la que se presenta al participante el estímulo no-verbal y se le pide responder a preguntas de carácter abierto. Candeias (2003) reportó una consistencia interna (alpha de Cronbach) de .95 y correlaciones significativas con otras pruebas de razonamiento social. La tarea está actualmente estandarizada para la población portuguesa.

Entrevista de Estrategias de Interacción con los Compañeros (CEIC) (Díaz-Aguado \& Royo, 1995). Tarea diseñada y baremada en España, forma parte de la batería de instrumentos propuesta por el Ministerio de Asuntos Sociales de España para evaluar competencia socioemocional en niños y adolescentes. Consiste en 4 situaciones basadas en el contexto escolar, en las que un personaje debe idear estrategias para solucionar un problema de tipo social (e.g., conseguir que un compañero le devuelva los materiales escolares que le quita y nunca le devuelve). El instrumento tiene dos versiones, una para niños y otra para adolescentes. Si bien ambas versiones constan de 4 estímulos (historias), éstas difieren entre sí. En ambos casos, se pide a los participantes definir cuál es el problema y qué harían para resolverlo, pero en el caso de los adolescentes, se añade una pregunta referida a la anticipación de las consecuencias. Los autores informan coeficientes alpha entre .51 y .72 para la versión de niños, y entre .85 y .90 para la versión de adolescentes.

La diferencia principal entre ambos instrumentos es que la Tarea PCIS presenta estímulos gráficos no-verbales y requiere que los participantes "armen" una historia (es decir, que definan el problema), mientras la Tarea CEIC presenta estímulos verbales en los que el problema está predefinido y corresponde a un conflicto interpersonal frecuente en la vida escolar.

\section{Procedimiento}

La recogida de datos se realizó entre los años 2005 y 2006. La aplicación se realizó de manera individual en los colegios, contando para ello con un lugar adecuado en términos de acústica y luminosidad. A los alumnos más pequeños (grupos 1 y 2 ) se les pidió que emitieran sus respuestas oralmente, las que fueron transcritas verbatim.

Las respuestas fueron transcritas y codificadas de acuerdo a los procedimientos de codificación propuestos originalmente por los autores. Los datos fueron analizados mediante el paquete estadístico SPSS 13.

\section{Resultados}

\section{Diferencias de Medias Entre Grupos}

\section{Tarea PCIS}

Los criterios originales son: (a) Comprensión del Problema: este criterio está compuesto por i) comprensión general del problema, ii) comprensión del carácter social del problema, y iii) percepción de las claves sociales de la situación; la suma de estos puntuaciones otorga una puntuación de "Comprensión total”; (b) Concepción de un plan, (c) Resolución del problema, y (d) Verificación del problema.

Efecto principal de nivel intelectual. Los resultados del ANOVA multivariante 2 (capacidad cognitiva: alta y media) x 5 (tramo de edad) indicaron diferencias significativas a favor del grupo ACC en todos los criterios: comprensión $(F(1.90)=17.128, p$ $<.001$, eta $\left.{ }^{2}=.160\right)$, concepción de un plan $(F(1.90)$ $=7.519, p<.01$, eta $\left.^{2}=.077\right)$, resolución $(F(1.90)=$ $\left.6.489, p<.05, e t a^{2}=.067\right)$, y verificación $(F(1.90)$ $=4.051, p<.05$, eta $\left.^{2}=.043\right)($ ver Tabla 1$)$. Para estudiar si hubo diferencias significativas en función de la capacidad cognitiva en todos los tramos de edad utilizamos la Prueba $t$ de Student con la corrección de Bonferroni ${ }^{3}$. Encontramos que, con un nivel de significación de .007, las diferencias significativas a favor del grupo con ACC se dieron en el criterio de comprensión entre los 12 y 17 años; en el de concepción de un plan entre los 9-11 y los 15-17 años; en el criterio de resolución entre los 9 y 11 años; y en el criterio de verificación entre los 15 y 17 años.

Efecto principal del grupo de edad. Encontramos diferencias significativas a favor de los grupos de mayor edad cronológica en todos los criterios: comprensión $\left(F(4.90)=12.442, p<.001\right.$, eta ${ }^{2}=$ $.356)$, concepción $\left(F(4.90)=14.154, p<.001\right.$ eta $^{2}$ $=.386)$, resolución $\left(F(4.90)=9.454, p<.001\right.$, eta ${ }^{2}=$ $.296)$, y verificación $(F(4.90)=14.182, p<.001$, $\left.e t a^{2}=.387\right)$ (ver Tabla 1). Mediante comparaciones post-hoc utilizando la prueba de Scheffé observamos que las diferencias en función de la edad se dieron en el grupo 1 (de 4-5 años), el que obtuvo puntuaciones significativamente más bajas que los demás grupos.

Efecto de interacción. Encontramos un efecto de interacción significativo entre la capacidad cognitiva y el tramo de edad, para el criterio de verificación de la respuesta $\left(F(4.90)=2.501, p<.05, e^{2} a^{2}=.100\right)$. Como se observa en la Tabla 1, mientras que en el grupo de capacidad habitual se observó un progresivo aumento en las puntuaciones en la verificación de la respuesta

\footnotetext{
El Método de Bonferroni controla la tasa de error dividiendo el nivel de significación entre el número de comparaciones llevadas a cabo (Pardo \& Ruiz, 2002). En nuestro caso, las diferencias entre pares de grupos dentro de cada tramo de edad son efectos simples de la interacción 2 x 5 . Esta interacción se puede descomponer en siete efectos simples diferentes. Por ello, utilizamos un nivel de significación corregido: $0.05 / 7=$ 0.007 .
} 
Tabla 1

Media (y desviación típica) de los puntajes obtenidos en la Tarea PCIS, según capacidad cognitiva, grupo de edad y criterios de codificación.

\begin{tabular}{|c|c|c|c|c|c|c|c|c|}
\hline & & & $\begin{array}{l}\text { Grupo } 1 \\
\text { (4-5 años) }\end{array}$ & $\begin{array}{l}\text { Grupo } 2 \\
\text { (6-8 años) }\end{array}$ & $\begin{array}{l}\text { Grupo } 3 \\
\text { (9-11 años) }\end{array}$ & $\begin{array}{l}\text { Grupo } 4 \\
\text { (12-14 años) }\end{array}$ & $\begin{array}{l}\text { Grupo } 5 \\
\text { (15-17 años) }\end{array}$ & Media Total \\
\hline \multirow{8}{*}{$\begin{array}{l}\text { Compresión } \\
\text { del } \\
\text { problema }\end{array}$} & \multirow[t]{2}{*}{$\mathrm{CG}$} & Alta & $2.50(1.08)$ & $3.70(1.49)$ & $4.00(1.15)$ & $4.70(1.06)$ & $6.00(0.00)$ & $4.18(1.56)^{* *}$ \\
\hline & & Media & $2.90(1.37)$ & $3.30(1.64)$ & $3.60(1.35)$ & $3.50(1.35)$ & $4.10(1.37)$ & $3.46(1.45)$ \\
\hline & \multirow[t]{2}{*}{$\mathrm{CS}$} & Alta & $2.90(1.45)$ & $4.00(1.41)$ & $5.10(1.66)$ & $5.70(1.57)$ & $5.80(1.23)$ & $4.70(1.80)^{* *}$ \\
\hline & & Media & $3.00(1.63)$ & $3.80(1.55)$ & $3.50(2.46)$ & $3.60(2.27)$ & $4.80(0.79)$ & $3.74(1.86)$ \\
\hline & \multirow[t]{2}{*}{$\mathrm{P}$} & Alta & $2.40(1.35)$ & $4.60(2.27)$ & $5.30(2.80)$ & $6.20(2.20)$ & $5.90(1.37)$ & $4.88(2.41)^{* * *}$ \\
\hline & & Media & $1.60(1.65)$ & $4.30(1.49)$ & $3.40(1.78)$ & $4.90(2.38)$ & $3.40(1.71)$ & $3.52(2.08)$ \\
\hline & \multirow[t]{2}{*}{ Total } & Alta & $7.80(2.90)$ & $12.30(3.02)$ & $14.40(4.60)$ & $16.60(3.10)$ & $17.70(1.89)$ & $13.76(4.70)^{* * *}$ \\
\hline & & Media & $7.50(3.95)$ & $11.40(3.78)$ & $10.50(4.81)$ & $12.00(4.81)$ & $12.30(2.26)$ & $10.74(4.24)$ \\
\hline \multirow{2}{*}{\multicolumn{2}{|c|}{$\begin{array}{l}\text { Concepción } \\
\text { de un plan }\end{array}$}} & Alta & $6.20(3.19)$ & $6.20(2.39)$ & $7.90(1.73)$ & $9.40(1.71)$ & $11.80(1.55)$ & $8.30(3.00)^{* *}$ \\
\hline & & Media & $4.60(2.22)$ & $7.30(2.41)$ & $6.00(1.76)$ & $8.60(2.88)$ & $8.70(2.54)$ & $7.04(2.78)$ \\
\hline \multirow{2}{*}{\multicolumn{2}{|c|}{$\begin{array}{l}\text { Resolución } \\
\text { del problema }\end{array}$}} & Alta & $5.60(2.63)$ & $5.70(2.54)$ & $9.50(2.17)$ & $9.50(2.59)$ & $9.10(2.13)$ & $7.88(2.97)^{* *}$ \\
\hline & & Media & $4.00(1.89)$ & $6.30(2.26)$ & $5.80(3.55)$ & $8.00(2.87)$ & $8.70(2.87)$ & $6.56(3.12)$ \\
\hline \multirow{2}{*}{\multicolumn{2}{|c|}{$\begin{array}{l}\text { Verificación } \\
\text { del problema }\end{array}$}} & Alta & $1.20(1.02)$ & $2.60(1.65)$ & $3.70(1.06)$ & $3.70(2.06)$ & $6.40(1.26)$ & $3.52(2.22)^{*}$ \\
\hline & & Media & $1.10(1.28)$ & $2.60(2.07)$ & $3.30(1.49)$ & $3.60(2.27)$ & $3.60(2.12)$ & $2.84(2.04)$ \\
\hline
\end{tabular}

Nota $: \mathrm{CG}=$ Comprensión general del problema; $\mathrm{CS}=$ Comprensión del carácter social; $\mathrm{P}=$ Percepción de las claves sociales; Total $=$ sumatoria $\mathrm{CG}+\mathrm{CS}+\mathrm{P}$

$* p<.05 * * p<.01 * * * p<.001$

con la edad, que tendió a estabilizarse a partir de los 12-14 años, en el grupo con ACC se observó un aumento brusco y significativo en la verificación de las respuestas a partir de los 15-17 años de edad.

\section{Tarea CEIC}

Los criterios para la versión CEIC-Niños son: (a) elaboración, (b) eficacia, (c) consecuencias sociales, y (d) asertividad. Los criterios para la versión CEIC-Adolescentes son: (a) orientación práctica, (b) definición del problema, (c) anticipación de las consecuencias, y (d) consecuencias sociales. Dado que la tarea es de respuesta libre, se analizaron las respuestas en función de la "respuesta total": el promedio entre la primera respuesta (Respuesta A: la primera solución planteada) y el resto de las respuestas (Respuesta B: el promedio de la segunda, tercera....n solución propuesta). Se realizó un ANOVA multivariante $2 \times 5$ para cada criterio de ambas versiones del instrumento.

Efecto principal de nivel intelectual. Utilizando los criterios de la versión CEIC-Niños $(n=60)$ no encontramos diferencias significativas en función de la capacidad cognitiva. Al analizar la versión CEICAdolescentes $(n=40)$ encontramos únicamente un efecto principal en el criterio de anticipación de las consecuencias $\left(F(1)=5.342, p<.05\right.$, eta $\left.{ }^{2}=.129\right)$. La prueba $t$ de Student con corrección de Bonferroni indicó que el grupo con ACC del tramo de edad 4 (12-14 años) obtuvo puntuaciones significativamente más altas que el grupo de capacidad media.

Efecto principal de edad. Utilizando los criterios para la versión CEIC-Niños encontramos diferencias significativas en función del grupo de edad, a favor de los grupos de mayor edad cronológica, en todos los criterios (elaboración: $F(4)=5.342, p<.001$, eta ${ }^{2}=.192$; eficacia: $F(4)=6.958, p<.001$, $e t a^{2}=$ .236 ; consecuencias: $F(4)=3.453, p<.05$, eta $^{2}=$ .133 ; asertividad: $F(4)=7.113, p<.001$, eta $^{2}=.240$ ). Mediante la prueba de Scheffé observamos que las diferencias se dieron entre el grupo 1 (4-5 años) respecto al grupo 3 (6-8 años); y entre el grupo 2 y el grupo 3 (9-11 años). En la versión CEIC-Adolescentes encontramos diferencias en función del grupo de edad a favor del grupo de mayor edad cronológica, únicamente en el subcriterio de definición del carácter social del problema $(F(4)=4.667, p<.05$, eta $\left.{ }^{2}=.037\right)($ ver Tabla 2).

Efecto de interacción. Encontramos, además, un efecto de interacción entre la capacidad cognitiva y el tramo de edad, para el criterio de anticipación de 
Tabla 2

Media (y desviación típica) de los puntajes obtenidos en la Tarea CEIC (versión niños y adolescentes) según capacidad cognitiva, grupo de edad y criterios de codificación.

\begin{tabular}{|c|c|c|c|c|c|c|c|c|}
\hline & & & $\begin{array}{l}\text { Grupo } 1 \\
\text { (4-5 años) }\end{array}$ & $\begin{array}{l}\text { Grupo } 2 \\
\text { (6-8 años) }\end{array}$ & $\begin{array}{l}\text { Grupo } 3 \\
\text { (9-11 años) }\end{array}$ & $\begin{array}{l}\text { Grupo } 4 \\
\text { (12-14 años) }\end{array}$ & $\begin{array}{l}\text { Grupo } 5 \\
\text { (15-17 años) }\end{array}$ & Media Total \\
\hline \multirow{8}{*}{ 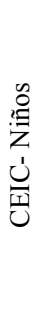 } & Elaboración & Alta & $8.35(3.39)$ & $9.60(1.88)$ & $10.80(1.84)$ & & & $9.58(2.60)$ \\
\hline & & Media & $8.05(3.62)$ & $7.55(2.72)$ & $11.55(2.22)$ & & & $9.05(3.34)$ \\
\hline & Eficacia & Alta & $8.80(3.62)$ & $8.75(1.57)$ & $10.05(1.73)$ & & & $9.20(2.48)$ \\
\hline & & Media & $7.70(3.37)$ & $7.40(2.31)$ & $9.70(1.81)$ & & & $8.27(2.61)$ \\
\hline & Consecuencias & Alta & $9.70(4.55)$ & $10.00(2.27)$ & $10.05(2.35)$ & & & $9.91(3.06)$ \\
\hline & & Media & $8.45(3.90)$ & $7.85(2.73)$ & $10.05(1.98)$ & & & $8.78(3.02)$ \\
\hline & Asertividad & Alta & $10.30(2.68)$ & $10.40(2.17)$ & $11.50(1.06)$ & & & $10.73(2.09)$ \\
\hline & & Media & $10.10(2.84)$ & $9.50(4.24)$ & $12.70(1.44)$ & & & $10.77(3.27)$ \\
\hline \multirow{8}{*}{ 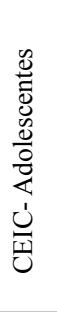 } & Orientación práctica & Alta & & & & $1.95(0.42)$ & $2.03(0.38)$ & $1.99(0.39)$ \\
\hline & & Media & & & & $1.95(0.33)$ & $1.98(0.58)$ & $1.96(0.46)$ \\
\hline & Definición del problema & Alta & & & & $2.03(0.46)$ & $1.95(0.63)$ & $1.99(0.55)$ \\
\hline & & Media & & & & $2.10(1.13)$ & $1.72(0.49)$ & $1.91(0.87)$ \\
\hline & Anticipación & Alta & & & & $2.22(0.36)$ & $1.95(0.43)$ & $2.08(0.41)^{*}$ \\
\hline & & Media & & & & $1.65(0.71)$ & $1.91(0.43)$ & $1.78(0.43)$ \\
\hline & Consecuencias & Alta & & & & $2.65(0.50)$ & $2.66(0.49)$ & $2.65(0.48)$ \\
\hline & & Media & & & & $2.16(0.57)$ & $2.71(0.45)$ & $2.44(0.58)$ \\
\hline
\end{tabular}

$* p<.05$

las consecuencias de la versión CEIC-Adolescentes $\left(F(1)=4.090, p<.05, e t a^{2}=.102\right)$. Mientras que las puntuaciones de los adolescentes con ACC no variaron al pasar del tramo de edad 12-14 años a 15-17 años, los adolescentes de capacidad media mejoraron su capacidad de anticipación de las consecuencias sociales entre estos tramos de edad (ver Tabla 2).

\section{Relaciones Entre Criterios y Variables}

El análisis de correlaciones bivariadas sobre la muestra total $(N=100)$ mostró que, de los dos instrumentos, sólo la Tarea PCIS correlacionó significativamente con el CI Total, CI Verbal y CI Manipulativo de la Escala de Wechsler $(r=.37, .34$ y .34 , respectivamente). La correlación entre la tarea PCIS y la tarea CEIC-Niños y CEIC-Adolescentes fue significativa aunque moderada $(r=.36$ y $r=.40$ respectivamente, $p<.001)$. Al controlar el efecto del CI Total y del CI Verbal, la relación entre las tareas PCIS y CEIC-Niños mantuvo su significación estadística $(r=.32 \mathrm{y} r$ $=.33$ respectivamente, $p<.01$ ) y la magnitud de la relación no cambió sustancialmente. En adolescentes, la magnitud de la correlación aumentó tras controlar el efecto del CI Total y Verbal $(r=.41, p<.05$ y $r=$ $.49, p<.01$, respectivamente).

En las Tablas 3 y 4 presentamos la matriz de intercorrelaciones entre los criterios de la Tarea PCIS y de la Tarea CEIC versión niños y adolescentes, respectivamente.

Los resultados de regresión múltiple (método stepwise) indicaron que, de todos los criterios de los dos instrumentos aplicados, sólo los criterios de "comprensión del problema" (PCIS) y "consecuencias sociales-niños" (CEIC) ingresaron al modelo predictivo sobre la puntuación en la Escala de Wechsler. La comprensión inicial del problema explicó el 32\% de la varianza en la puntuación CI Total $\left(R^{2}=.321, F(1,36)=16.991, p<.001\right)$ y la adecuación de solución planteada explicó un $14 \%$ de varianza adicional $\left(\Delta R^{2}=.141, F(1,35)=\right.$ $15.015, p<.001)$. A su vez, de todos los criterios estudiados, sólo la ideación de un plan de acción (PCIS) tuvo un valor predictivo sobre la edad de los participantes, explicando el $12 \%$ de la varianza del factor edad cronológica $\left(R^{2}=.122, F(1,38)=\right.$ $5.262, p<.05)$.

\section{Discusión}

De los datos anteriores podemos concluir que los alumnos con ACC presentan un mejor desempeño en la tarea de inteligencia social (Candeias, 2003, 2005) y un desempeño menos claro en la tarea de competencia socioemocional (Díaz-Aguado \& Royo, 1995), en relación a sus pares de capacidad cognitiva media. Mientras que, a partir de los 9-11 
Tabla 3

Matriz de intercorrelaciones entre los criterios de la Tarea PCIS y de la Tarea CEIC-Niños

\begin{tabular}{|c|c|c|c|c|c|c|c|c|}
\hline Criterio & 1 CEIC) & 2 CEIC) & 3 (CEIC) & 4 CEIC) & 5 (PCIS) & 6 (PCIS) & 7 PCIS) & 8 PCIS) \\
\hline 1.Elaboración (CEIC) & - & & & & & & & \\
\hline 2. Eficacia (CEIC) & $.83 * * *$ & - & & & & & & \\
\hline 3. Consecuencias (CEIC) & $.83 * * *$ & $.94 * * *$ & - & & & & & \\
\hline 4. Asertividad (CEIC) & $.56 * * *$ & $.46 * * *$ & $.43 * * *$ & - & & & & \\
\hline 5. Comprensión (PCIS) & $.24 *$ & $.27 * *$ & $.24 *$ & $.30^{* *}$ & - & & & \\
\hline 6. Concepción plan (PCIS) & $.23 *$ & $.32 * *$ & $.29 * *$ & $.21^{*}$ & $.54 * * *$ & - & & \\
\hline 7. Resolución (PCIS) & $.23^{*}$ & $.32 * *$ & $.25^{*}$ & $.26^{* *}$ & $.63 * * *$ & $.66 * * *$ & - & \\
\hline 8. Verificación (PCIS) & $.24 *$ & $.29 * *$ & .19 & $.29 * *$ & $.57 * * *$ & $.57 * * *$ & $.60 * * *$ & - \\
\hline
\end{tabular}

$* p<.05 * * p<.01 * * * p<.001$

Tabla 4

Matriz de intercorrelaciones entre los criterios de la Tarea PCIS y de la Tarea CEIC-Adolescentes.

\begin{tabular}{|c|c|c|c|c|c|c|c|c|}
\hline Criterio & 1 (CEIC) & 2 (CEIC) & 3 (CEIC) & 4 (CEIC) & 5 (PCIS) & 6 (PCIS) & 7 (PCIS) & 8 (PCIS) \\
\hline 1.Orientación práctica (CEIC) & - & & & & & & & \\
\hline 2. Definición (CEIC) & .14 & - & & & & & & \\
\hline 3. Anticipación (CEIC) & .14 & .17 & - & & & & & \\
\hline 4. Consecuencias (CEIC) & -.06 & $.45^{* *}$ & $.51 * * *$ & - & & & & \\
\hline 5. Comprensión (PCIS) & .26 & .21 & $.30 *$ & .09 & - & & & \\
\hline 6. Concepción plan (PCIS) & .05 & .23 & .27 & -.06 & $.54 * * *$ & - & & \\
\hline 7. Resolución (PCIS) & .24 & .24 & $.48^{* *}$ & .17 & $.62 * * *$ & $.65 * * *$ & - & \\
\hline 8. Verificación (PCIS) & .17 & $.31 *$ & $.44 * *$ & $.34 *$ & $.57 * * *$ & $.57 * * *$ & $.60 * * *$ & - \\
\hline
\end{tabular}

$* p<.05 * * p<.01 * * * p<.001$

años, su desempeño en la Tarea PCIS es superior a sus pares en todos los criterios (comprensión del problema, concepción de un plan de acción, resolución del problema y verificación de ésta), su desempeño en la Tarea CEIC es superior únicamente en el criterio de anticipación de las consecuencias en los adolescentes.

La diferencia entre ambas tareas es fundamentalmente que la PCIS exige no sólo la aplicación de estrategias de interacción social, sino también la definición de la situación-problema. Los resultados análisis de regresión múltiple sugieren que, de todos los criterios estudiados, es el comprender y definir inicialmente la situación social lo que se relaciona de manera más estrecha con la capacidad cognitiva general.

A continuación discutimos estos resultados a la luz de algunos debates recogidos en la literatura con respecto de la inteligencia social.

\section{¿Inteligencia General o Inteligencia Social?}

Los resultados nos permiten concluir que los niños con ACC sí presentan mayor habilidad para idear estrategias cognitivas para la resolución de problemas de orden social, aunque no difieran de sus pares en su competencia socioemocional, es decir, en su capacidad para elaborar estrategias adecuadas de interacción con sus compañeros frente a situaciones de interacción 
que requieren de cooperación y negociación. Un patrón similar se observa en los adolescentes con ACC, quienes únicamente presentaron mayor capacidad para anticipar las consecuencias sociales de las estrategias de interacción social que proponen.

Los resultados del análisis correlacional efectuado sobre la muestra total indican que, de hecho, la tarea de inteligencia social guarda una relación significativa con el CI Verbal, de Ejecución y Total de la Escala de Wechsler. Esto no se observa en el caso de la tarea de competencia social. Y sin embargo, hay una relación significativa entre las medidas de inteligencia y competencia social. Esto podría estar indicando que aquellos aspectos de la Tarea PCIS que no son compartidos por el CI, son sin embargo compartidos por la Tarea CEIC. Estos resultados apoyarían la tesis de una relación parcial entre la capacidad cognitiva general y la inteligencia social. Consecuentemente, los datos entregan soporte a la noción de la inteligencia social como un constructo de relativa independencia con respecto de la inteligencia general (Gardner, 1983). Si bien hay una parte de la inteligencia social que viene explicada por procesos cognitivos generales, éstos quizás no logren explicar toda la varianza en las medidas de inteligencia social.

Pero los resultados también revelan el carácter multidimensional de la inteligencia social, lo que hace difícil su medición. De esto ya se habían dado cuenta los primeros investigadores que buscaron crear medidas de inteligencia social (Hendricks, Guilford \& Hoepfner, 1969; Hunt, 1928; Moss \& Hunt, 1927; O'Sullivan, Guilford \& deMille, 1965). En este estudio, constatamos que la inteligencia social de niños y adolescentes con ACC depende del tipo de instrumento que se utilice. La medida más "cognitiva" de inteligencia social presentó diferencias significativas a favor del grupo con altas capacidades, pero la medida centrada en la aplicación de estrategias específicas no mostró diferencias (salvo en la anticipación de las consecuencias sociales, que podemos considerar un aspecto más "cognitivo" del procesamiento). Estos resultados parecen estar indicando que el constructo de inteligencia social tiene, por lo menos, dos dimensiones: una referida a las estrategias de decodificación y codificación de la información de contenido social, y otra referida al contenido de los discursos ideados para resolver problemas de tipo social. Quizás debido a que la Tarea PCIS y la Tarea CEIC miden en lo principal cada dimensión, respectivamente, es que los resultados de su aplicación en una misma muestra difieren.
Por otra parte, es importante destacar que los niños y adolescentes no presentaron un desempeño menor que sus pares, en ningún criterio de las dos tareas. Estos resultados avalan la tesis de una "normalidad socioemocional" de las personas con altas capacidades durante su infancia y adolescencia (Gross, 2000; Terman, 1925/1947; Terman \& Oden, 1947). La pregunta no es, entonces, si estos niños tienen una menor inteligencia social, sino que por el contrario, si podemos sostener que presenten en algunos aspectos una mayor inteligencia social. Al respecto, los resultados de este estudio sugieren que los procesos cognitivos generales actúan como facilitadores de los procesos de dominio específico referidos a las personas y su mundo social, en tanto permiten a los adolescentes con ACC desarrollar estrategias de decodificación social más precisas. Queda aún por estudiar si ellos son, además, más concientes de estos procesos.

\section{¿Inteligencia o Competencia Social?}

Es necesario considerar que las dos medidas empleadas en este estudio piden a los participantes "pensar" sobre el mundo social. Cabe preguntarse si estas medidas de "razonamiento social" guardan o no una relación directa con medidas globales de aceptación social. Es decir, si el "pensar sobre" se traduce de manera automática en el "actuar en". Quizás allí radique la diferencia entre inteligencia y competencia social.

Para Goleman (2006) la inteligencia social se compone de dos aspectos distintivos: la conciencia social y la aptitud social. Mientras que el primero se refiere a la comprensión y el sentimiento por los demás (e incluye, según él, la empatía o la interpretación de las señales emocionales no verbales; la sintonía o escucha receptiva; la exactitud empática o comprensión de los pensamientos, sentimientos e intenciones de los demás; y la cognición social o comprensión del funcionamiento del mundo social), la aptitud social apunta a la aplicación de la conciencia social en la vida cotidiana. Esta distinción marca la misma diferencia que ya sugieren los datos: que el "saber hacer" es distinto al "hacer".

\footnotetext{
4 De hecho, nuestros datos indican que la mayor capacidad para inferir estados mentales epistémicos y emocionales, básicos y avanzados (parte de lo que Goleman llamaría “conciencia social"), así como el mejor uso de estrategias de planificación, ejecución y verificación de problemas sociales (que hemos presentado en este artículo, y que también formarían parte de la "conciencia social") tienen escaso valor predictivo sobre medidas globales de aceptación social (López y Sotillo, en preparación).
} 
Por último, quisiera discutir el uso de los términos de "inteligencia" y "competencia" para hablar del razonamiento sobre contenidos sociales. El término "inteligencia" tiene una carga semántica que históricamente ha remitido a una concepción de la mente humana como genéticamente determinada, estable y de relativa permeabilidad al medio cultural. En el caso de la inteligencia social, es poco probable que la influencia ambiental ejerza poco peso en la variación intra- e inter-individual sobre el razonamiento social. Más bien, se esperaría lo contrario. Las experiencias cotidianas con hermanos mayores, con compañeros de curso y con adultos probablemente incidan de manera muy significativa en la mayor o menor "inteligencia social" que desarrolle una persona. Por otra parte, la palabra "competencia" alude a una habilidad que se adquiere y desarrolla a través de la experiencia, y por tanto tiene una carga más cultural. En contextos de intervención (por ejemplo, dentro del ámbito educativo) merece la pena preguntarse cuál término conviene utilizar. Hablar de "competencias socioemocionales" implica entregar al profesor y a los adultos significativos la tarea de enseñar y entregar contextos que permitan desarrollar estas habilidades (Milicic, comunicación personal). Claramente, las posibilidades de intervención son distintas si hablamos de una "capacidad instalada", que de un "dominio a aprender".

\section{Referencias}

Binet, A., Simon, T. \& Kite, E. (1916). The intelligence of the feeble-minded. Baltimore, MD: Williams and Wilkins Co.

Bralic, S. \& Romagnoli, C. (2000). Niños y jóvenes con talentos: Un desafio para Chile. Santiago: Dolmen.

Candeias, M. A. (2003). Prova Cognitiva de Inteligencia Social. En M. Gonçalves, M. Simões, A. Almeida \& C. Machado (Coords.), Avaliação psicológica: Instrumentos validados para a população portuguesa (pp. 179-196). Coimbra: Quarteto Editora.

Candeias, M. A. (2005). Prova Cognitiva de Inteligencia Social: Manual. Manuscrito no publicado. Evora: Universidad de Evora.

Casillas, M. A. (1996). El desarrollo de potenciales en la educación preescolar. Revista Renglones, 6, 17-21.

Cross, T. L. \& Coleman, L. J. (1993). The social cognition of gifted adolescents: An exploration of the stigma of giftedness. Roeper Review, 16(1), 37-40.

Cross, T. L., Coleman, L. J. \& Stewart, R. A. (1995). Psychosocial diversity among gifted adolescents: An exploratory study of two groups. Roeper Review, 17(3), 181-185.

Cupertino, C. M. \& Ancona-López, M. (1992). Brazilian middle class gifted students and their perceptions of leadership role. En F. J. Mönks y W. A. Peters (Eds.), Talent for the future: Social and personality development of gifted children. Proceedings of the Ninth World Conference on Gifted and Talented Children (pp. 110-116). Assen, Netherlands: Van Gorcum and Co.
Díaz-Aguado, M. J. \& Royo, P. (1995). La evaluación de la competencia socioemocional a través de una entrevista semiestructurada. En M. J. Díaz-Aguado (Dir.), Niños con dificultades socioemocionales: Instrumentos de evaluación. Madrid: Ministerio de Asuntos Sociales.

Freeman, J. (1998). Aspectos emocionales de la sobredotación. En J. Freeman (Ed.), Los niños superdotados. Aspectos psicológicos y pedagógicos. Madrid: Santillana.

Gagné, F. (1993). Constructs and models pertaining to exceptional human abilities. En K. A. Heller, F. J. Mönks y A. H. Passow (Eds.), International handbook of research and development of giftedness and talent (pp. 63-85). Oxford: Pergamon Press.

Gardner, H. (1983). Frames of mind: The theory of multiple intelligences. New York: Basic Books.

Gardner, H. (2000). The giftedness matrix: A developmental perspective. En R. Friedman y B. Shore (Eds.), Talents unfolding: Cognition and development (pp. 77-88). Washington, DC: American Psychological Association.

George, D. (1992). Gifted education in England. Roeper Review, 14(4), 201-204.

Goleman, D. (2006). La inteligencia social. Barcelona: Kairós.

Gross, M. U. (2000). Issues in the cognitive development of exceptionally and profoundly gifted individuals. En K. A. Heller, F. J. Monks, R. Sternberg y R. F. Subotnik (Eds.), International handbook of giftedness and talent (pp. 179-192). Amsterdam: Elsevier.

Guignard, J. H. \& Zenasni, F. F. (2004). Les caractéristiques émotionnelles des enfants a haut potentiel. Psychologie Française, 49(3), 305-319.

Guilford, J. P. (1967). The nature of intelligence. New York: McGraw Hill.

Hendricks, M., Guilford, J. P., \& Hoepfner, R. (1969). Measuring creative social intelligence. Reports from the Psychological Laboratory, University of Southern California, 42.

Hunt, T. (1928). The measurement of social intelligence. Journal of Applied Psychology, 12, 317-334.

Jensen, A. R. (2002). Psychometric G: Definition and substantiation. En R. J. Sternberg, y E. L. Grigorenko (Eds.), The general factor of intelligence: How general is it? (pp. 39-53). Mahwah, NJ: Lawrence Erlbaum.

Jiménez Fernández, C. (2004). Alumnos superdotados: Caracterización e identificación. En Secretaría General de Educación (Ed.), Diagnóstico y atención a los alumnos con necesidades educativas especificas: Alumnos intelectualmente superdotados (pp. 1-25). Madrid: Ministerio de Educación y Ciencia.

Kanevsky, L. S. (1994). A comparative study of children's learning in the zone of proximal development. European Journal for High Ability, 5(2), 163-175.

Kihlstrom, J. F. \& Cantor, N. (2000). Social intelligence. En R. J. Sternberg (Ed.), Handbook of intelligence, $2^{\mathrm{a}}$ ed. (pp. 359379). Cambridge.: Cambridge University Press.

Lubinski, D. (2004). Introduction to the special section on cognitive abilities: 100 years after Spearman's (1904) "general intelligence, objectively determined and measured". Journal of Personality and Social Psychology, 86(1), 96-111.

Marland, S. P. (1972). Education of the gifted and talented. Report to the Congress of the United States by the U.S. Commissioner of Education. Washington, DC: U.S. Government Printing Office.

Morelock, M. J. (2000). A sociohistorical perspective on exceptionally high-IQ children. En R. C. Friedman y B. M. Shore (Eds.), Talents unfolding: Cognition and development (pp. 55-75). Washington, DC: American Psychological Press. 
Moss, F. A., \& Hunt, T. (1927). Are you socially intelligent? Scientific American, 137, 108-110.

O'Sullivan, M., Guilford, J. P., \& de Mille, R. (1965). The measurement of social intelligence. Reports from the Psychological Laboratory, University of Southern California, 34.

Pardo, A. \& Ruiz, M. A. (2002). SPSS 11: Guía para el análisis de datos. Madrid: McGraw Hill.

Perleth, C. \& Heller, K. A. (1994). The Munich longitudinal study of giftedness. En B. Subotnick y K. D. Arnold (Eds.), Beyond Terman: Contemporary longitudinal studies of giftedness and talent. Norwood, NJ: Ablen Publishing Co.

Piechowski, M. M. \& Colangelo, N. (2004). Developmental potential of the gifted. En R. J. Sternberg (Ed.), Definitions and conceptions of giftedness: Essential readings in gifted education (pp. 117-132). Thousand Oaks, CA: Corwin Press.

Powell, P. M. (1980). Advanced social role-taking and cognitive development in gifted adults. International Journal of Aging and Human Development, 11(3), 177-192.

Renzulli, J. S. (1994). El concepto de los tres anillos de la superdotación: Un modelo de desarrollo para una productividad creativa. En Y. Benito (Ed.), Intervención e investigación psicoeducativas en alumnos superdotados. Salamanca: Amarú.

Rimm, S. B. \& Rimm-Kaufman, S. (2000). How Jane won: Profiles of successful women. New York: Crown.

Roeper, A. (1982). How the gifted cope with their emotions. Roeper Review, 5, 21-24.

Rogers, K. B. (1986). Do the gifted think and learn differently? A review of recent research and its implications for instruction. Journal for the Education of the Gifted, 10(1), 17-39.

Rosas, R., Boetto, C. \& Jordán, V. (1999). Introducción a la psicología de la inteligencia. Santiago de Chile: Ediciones Universidad Católica de Chile.

Shore, B. M. \& Kanevsky, L. S. (1993). Processes: Being and becoming gifted. En K. H. Heller, F. J. Monks \& A. H. Passow (Eds.), International handbook of research into giftedness and talent. Oxford: Pergamon Press.

Silverman, L. K. (1993). Counseling the gifted and talented. Denver, Co: Love Publishing Co.

Spearman, C. (1904). "General intelligence", objectively determined and measured. The American Journal of Psychology, 15, 201-293.

Spearman, C. (1927). The abilities of man. New York: MacMillan.

Sternberg, R. J. (1984). Toward a triarchic theory of human intelligence. Behavioral and Brain Sciences, 7, 269-287.
Sternberg, R. J. (2005). The theory of successful intelligence. Revista Interamericana de Psicología, 39(2), 189-202.

Sternberg, R. J. \& Barnes, M. (Eds.). (1988). The psychology of love. New Haven: Yale University Press

Sternberg, R. J. \& Wagner, R. (Eds.). (1986). Practical intelligence: Nature and origins of competence in the everyday world. Cambridge, U.K.: Cambridge University Press.

Sternberg, R. \& Wagner, R. (1989). The fate of the trait: A reply to Cantor and Khilstrom. En R. Wyer \& T. Scrull (Eds.), Social intelligence and cognitive assessments of personality (pp. 175-186). New Jersey: LEA.

Swiatek, M. A. \& Dorr, R. M. (1998). Revision of the Social Coping Questionnaire: Replication and extension of previous findings. Journal of Secondary Gifted Education, 10, 22-59.

Terman, L. M. (1925/1947). Genetic studies of genius. Stanford, CA: Stanford University Press.

Terman, L. M. \& Oden, M. (1947). The gifted child grows up: Twenty-Five year's follow-up of a superior group. California: Stanford University Press.

Terrasier, J. C. (1992). La disincronía. Creatividad y rigidez de la escuela frente al derecho a la diversidad. En Y. Benito (Ed.), Desarrollo y educación de los niños superdotados. Salamanca: Amarú.

Thorndike, E. L. (1920). Intelligence and its use. Harper's Magazine, 140, 227-235.

Thorndike, R. L. \& Stein, S. (1937). An evaluation of the attempts to measure social intelligence. Psychological Bulletin, $34,275-285$.

Thurstone, L. L. (1938). Primary mental abilities. Psychometric Monographs, 1, 1-121.

Van Tassel-Baska, J. (1998). Excellence in education: Gifted and talented learners. Denver, CO: Love Publishing Co.

Vernon, P. E. (1933). Some characteristics of the good judge of personality. Journal of Social Psychology, 4, 42-57.

Wechsler, D. (1939/1958). The measurement and appraisal of adult intelligence ( $4^{\text {th }}$ edition). Baltimore: Williams $y$ Wilkins.

Wechsler, D. (1974). Selected papers of David Wechsler. Oxford: Academic Press.

Winner, E. (2000). The origins and ends of giftedness. American Psychologist, 55(1), 159-169.

Yeates, K. O. \& Selman, R. L. (1989). Social competence in the schools: Toward an integrative developmental model for intervention. Developmental Review, 9, 64-100.

Fecha de recepción: junio de 2007.

Fecha de aceptación: octubre de 2007. 